\title{
Lexis
}

Journal in English Lexicology

Book reviews | 2007

\section{David CRYSTAL, Language and the Internet}

Cambridge University Press, 2007, 318 pages

\section{Graham Ranger}

\section{(2) OpenEdition}

\section{Journals}

\section{Electronic version}

URL: https://journals.openedition.org/lexis/1831

DOI: 10.4000/lexis.1831

ISSN: 1951-6215

\section{Publisher}

Université Jean Moulin - Lyon 3

\section{Electronic reference}

Graham Ranger, "David crystaL, Language and the Internet", Lexis [Online], Book reviews, Online since 29 August 2007, connection on 08 December 2022. URL: http://journals.openedition.org/lexis/1831 ; DOI: https://doi.org/10.4000/lexis.1831

This text was automatically generated on 29 September 2020.

\section{(c) $($ ) $(9)$}

Creative Commons - Attribution-NonCommercial-NoDerivatives 4.0 International - CC BY-NC-ND 4.0 https://creativecommons.org/licenses/by-nc-nd/4.0/ 


\title{
David CRYSTAL, Language and the Internet
}

Cambridge University Press, 2007, 318 pages

\author{
Graham Ranger
}

\section{REFERENCES}

David Crystal

Language and the Internet. Cambridge University Press, 2006. ISBN: 978-0521868594,

Prix : 34,10€, 318 pages

1 In the preface to Language and the Internet David Crystal explains how the shifting emphasis from technology to "people and purposes" (x) contributes to making the Internet a linguistic revolution. The aim of Language and the Internet is "to find out about the role of language in the Internet and the effect of the Internet on language" (x) and this objective is pursued over nine well-balanced chapters, one more than the first edition of the same book. Chapter One provides a general introduction and distinguishes seve" different linguistic situations found on the Internet. Chapters Two and Three look at linguistic features common to all seven situations, while chapters Four to Nine focus more precisely on the specific defining characteristics of each.

2 In Chapter One A Linguistic Perspective Crystal begins by asking whether the Internet constitutes a homogeneous language-using electronic situation. The answer is that it does not, and this in turn leads him to ask how many linguistic varieties we can in fact distinguish. In order to identify a variety of language, we first need to define our criteria. Crystal lists five important stylistic features of written language: graphic (visual presentation), orthographic, grammatical, lexical and discursive ("the structural organization of a text [9]"). To these he adds the phonetic and phonological features of spoken language. The Internet is essentially text-based at present, but, Crystal suggests, we can easily imagine speech becoming progressively more important as speech production and recognition techniques progress. These features help us to 
distinguish "seven broad Internet-using situations" (10) whose language is "significantly distinctive" (10): email, chatgroups (which exist in two varieties: synchronous and asynchronous), virtual worlds (or MUDs for Multi-User Domains), the World Wide Web, instant messaging and blogging (a recent appearance which has justified an additional chapter). In each of these situations things are still very much in development: "the only thing that is clear is that people are unclear about what is going to happen" (16). This transitory state makes the Internet a particularly fascinating linguistic arena for the linguist as "people are still getting to grips with the communicative potential made available to them" (16). The chapter continues by looking at how the language of Netspeak is spilling over into non-Internet situations. My own favourites are "He's 404" used to mean that someone is not around (21) or Crystal's comments on the exponential increase in the use of Internet-inspired prefixes (from e-mail to $e$-book, $e$-therapy...), suffixes (from .com to the dotcom crisis) and infixes (the ubiquitous @).

Chapter Two The medium of Netspeak begins by mentioning two fundamental constraints on the nature of Internet language: the keyboard and the screen. "There are," Crystal tells us, "certain traditional linguistic activities that this medium can facilitate very well, and others that it cannot handle at all [...] The heart of the matter seems to be [Netspeak's] relationship to written and spoken language" (23). Internet style manuals recommend to "write the way people talk" (27) but how far is this actually possible? The question leads Crystal to focus his attention first on the differences between spoken and written language. "Speech is typically time-bound, spontaneous, face-toface, socially interactive, loosely structured, immediately revisable, and prosodically rich. Writing is typically space-bound, contrived, visually decontextualized, factually communicative, elaborately structured, repeatedly revisable, and graphically rich" (31). ${ }^{1}$ Crucially, Netspeak "relies on characteristics belonging to both sides of the speech/ writing divide" (31). Webpages, for example, obviously tend towards written language while virtual worlds, synchronous chatgroups or instant messaging evoke the characteristics of spoken language. Even these last varieties are still very different from genuine speech. As Crystal points out, technological constraints mean that, unlike a spoken conversation, messages cannot overlap (33), the phenomenon of lag (slow and variable transfer speeds) makes the rhythm of an Internet "chat" slower than that of normal speech and often renders standard turn-taking procedures irrelevant. Netspeak lacks prosody or paralanguage, a lack which is compensated for by a range of linguistic strategies: repeated letters (hiiiiii, ooops... [37]), capitalization, smileys (a comprehensive table of examples is given), etc. Particularly interesting is the way how, in some Internet situations, people comment upon their own linguistic expression. Crystal gives the example of one character, Hoppy, who in the course of an exchange comments "<Hoppy giggles quietly to himself $>$ " (38). "These features of Netspeak have evolved as a way of avoiding the ambiguities and misperceptions which come when written language is made to carry the burden of speech" (42). A table indicating the presence or absence of the seven distinguishing features of spoken and written language in the six varieties of Netspeak (both types of chatgroups are dealt with together) allows the author to conclude that "on the whole, Netspeak is better seen as written language which has been pulled some way in the direction of speech than as spoken language which has been written down" (51). He suggests that it is, however, more helpful to think of Netspeak as something different: "a genuine 'third medium'" (52). 
Crystal next considers Netspeak in the light of Grice's conversational maxims. ${ }^{2}$ Things are particularly interesting here. The anonymity of the participants, and the requirement, particularly low-speed connections, to be brief, place considerable pressure on the maxims of Quality, Relevance, Quantity and Manner, as the various practices of spoofing, trolling, lurking or flaming described by Crystal testify!

In Chapter Three, Crystal moves on to the question of usage or Netiquette, as it is sometimes called. There is apparently a strongly felt need for linguistic guidance, due perhaps to the newness of the medium and the potential for disaster (in the form of flaming, for example) and "An interesting kind of semi-prescriptivism has begun to emerge, as a result" (67). However, a comparison of two usage guides, Wired Style and Cyberspeak reveals big differences in the apprehension of what is appropriate for Netspeak. One of the motors of the linguistic inventiveness of Netspeak is, Crystal suggests, to be found in its hacker origins: "Hackers are plainly very aware of their identity as members of an Internet culture (more precisely, a collection of subcultures), dating from the earliest days, proud of their common background and values, and conscious of their expertise. Most of the style manuals include a characterization of the hacker mindset and skills" (73). In this context, many sites incorporate FAQ's to help with etiquette while chatgroups or MUDs call upon moderators (or "wizards") to ensure that, linguistically speaking, things are running smoothly. The resulting linguistic situation is something of an oddity. "Such explicit guidance is unusual in the real world" (76), Crystal reminds us, but in a chatgroup a moderator may tell someone they are off-topic or even enforce various orders of punishment on a seasoned offender! There then follows a discussion of the principles underlying style guides: principles which can be those of "an unhelpful prescriptivism [...] similar in its naivety, unreality and oversimplication to that encountered in the grammar books of old" (78). Crystal argues rather, as we would expect, for a descriptive recognition of the considerable linguistic diversity of the Internet. The chapter concludes with a section entitled "Some features of Netspeak", listing the most salient distinctive linguistic characteristics of Netspeak from a descriptive perspective. These features may concern the lexicon (virus, net.police, HTML, THX), typography (a VERY important point, AltaVista), spelling (gamez, phreak), punctuation (minimal or repetitive, e.g. !!!) as well as the adoption of many symbols from programming code into more general use (!interesting = 'not interesting'). The examples provided are comprehensive and fascinating. Crystal ends his descriptive account of Netspeak as a whole by acknowledging that the speed at which things are changing makes it "difficult to be definitive about the variety's characteristics [...] Doubtless some of the linguistic features described above will still be contributing to Netspeak's identity in fifty years' time; others may not last another year" (98).

6 The following chapters of Language and the Internet deal with the different varieties of Netspeak individually. Chapter Four is entitled The language of e-mail. Crystal begins by looking at the "structural elements" of an e-mail, including the different elements of the header and the greetings and farewells. In dealing with the body of the message, Crystal turns to the recommendations of e-mail style guides which tend, as he notes, to be very similar to older guides of how to write traditional letters. One frequent piece of advice is that messages should be concise, ideally readable without scrolling down the screen, and an analysis of typical paragraph length appears to confirm this. Unlike traditional letters, emails also rely more heavily on exchange, allowing replies within the message in the form of line-by-line annotations, so that both (or all) authors 
receive a perfect copy of the state of the correspondence. This "framed message" is, as Crystal points out "a most unusual object, not like anything else in language use" (125). Again, unlike a written letter, an e-mail does not necessarily imply a format whereas traditional correspondence involves a "choice of notepaper, letterhead typography, style of paragraphing, and signature format" (126). In an e-mail content is all important, and the above features are absent. Even attempts to personalize e-mails through choice of background colour etc. may be freely ignored by the recipient's software. E-mails typically include more questions (including rhetorical questions) than traditional letters (127). Smileys and abbreviations of the LOL type are, however, far less prevalent than is often claimed, Crystal affirms. The chapter ends by looking at some of the limitations of the e-mail in comparison with the letter, or the telephone call. Crystal's general view is that too much attention has been drawn to the informal qualities of e-mails but that, "as the medium matures, it is becoming apparent that it is not exclusively an informal medium, and received opinion is going to have to change" (133).

7 In Chapter Five, The language of chatgroups, Crystal focusses on chatgroups, discussion forums and other similar "multi-participant electronic discussion" (134). He begins by looking at asynchronous chatgroups as these are apparently less innovative, linguistically speaking, than their synchronous counterparts. Studies of chatgroups have identified a number of "functional macrosegments" including "an introduction, a contentful message body, and a close" (cit. Herring 149)3. There is some unpredictability in subject matter, but moderators generally ensure that messages remain relevant to a given "thread". Although the diversity of such groups makes generalisation difficult, Crystal nonetheless characterises the language of asynchronous chat as "a mixture of informal letter and essay" (154). Synchronous chatgroups are, in linguistic terms, somewhat more revolutionary. After some technical considerations on the types of protocol and software available for participation in synchronous chat, Crystal compares such groups to real-life chat and concludes that the two are very different indeed. In synchronous chatgroups "There is a permanent shifting of the goalposts [...] the order in which messages arrive is governed by factors completely outside the control of the participants, such as the speed of their computers and the processing capacities of the service-providers. None of this makes for a 'conversation' in the conventional sense of the term." (161). Interventions are typically very short (one study notes that $80 \%$ of contributions are less than 5 words long) and contributors are identified by their nicknames (or "nicks") the study of which "is going to provide onomastics with a fascinating domain in due course" (166). "Wordplay is ubiquitous", Crystal notes, through use of rebus, abbreviations, elisions and nonce formations (one typical example: what a unifreakinversitynerd). Even a misspelling may "have privileged status in one group [since] Each group has its own history and a group memory exists [...] and is respected" (171). I found Crystal's comments on the anonymity of the medium particularly interesting: "it is important to note that, when participants are anonymous, the language of the interaction, as presented on screen, is all other group members have to go on. Subconsciously, at least, participants will be paying special attention to everyone's choice of words, nuances of phrasing, and other points of content and presentation" (172). In a final paragraph entitled Why chat? Crystal considers the reasons that lead people to chat on line. "If you had said to me, a few years ago, that it was possible to have a successful conversation while disregarding the standard conventions of turn-taking, logical sequence, time ordering, and the like, I 
would have been totally dismissive. But the evidence is clear: millions are doing just that." (176). The reason, Crystal suggests, is that, for many, the social advantages of the activity must outweigh the semantic disadvantages.

Chapter Six, The language of virtual worlds, moves on to the linguistic characteristics of the virtual worlds (or MUDs) of gamers. Unlike the other linguistic situations evoked so far, here the subject matter is imaginary. Gamers communicate (and play the game) by means of text commands, the two most important of which are saying and emoting. There also exist gag commands (to silence a player) or mutter and whisper commands (to exclude certain players from a conversation). "The narrative style of emotes gives a somewhat literary flavour to the interaction, which sits oddly alongside the often highly colloquial tone of direct speech" (189), notes Crystal. If, like me, you are a little unsure what is involved, here is a taster in one sequence quoted by Crystal as it would appear on the screen:

lynn says, 'leggo my Lego Tom'

Bunny eyes Ray warily.

lynn [to Penfold]: hrmph

Ray puts the annoying electronic bell in the Christmas tree. (189-190)

In conclusion Crystal decides that "MUDs operate in a curious Alice-like world" (198), quoting Cherny's remark that "understanding MUD culture requires understanding the ambivalence of MUDders toward their texts, which remain poised between the transience of speech and the persistence of documents" (199). ${ }^{4}$

Chapter Seven brings us back to more familiar ground with The language of the Web. The Web includes and combines all varieties of written or printed text. It is "graphically more eclectic than any domain of written language in the real world" (205) since a web page can include linear and non-linear text, flashing text, lists etc. Especially important is the hypertext link: "the most fundamental structural property of the Web, without which the medium would not exist" (210). The Web is evolving from a fairly static entity, created by a web designer and consulted by a web user, into a more dynamic phenomenon, as possibilities for interaction are increasingly built into web pages. Crystal devotes several pages to the problem of intelligently sorting the increasingly numerous answers to simple websearches by means of a "semantic web", quoting Berners-Lee. "The aim of the SW is to "develop languages for expressing information in a machine processable form'" (219). ${ }^{5}$ Crystal is critical of the project and what he calls the "linguistic innocence" (220) of the taxonomies proposed which might just reflect "the programmer's prescriptive usage preferences" (225), concluding that there is considerable potential for linguistic tools in the elaboration of relevant search algorithms. The chapter ends with an interesting final section entitled Languages on the Web that looks at the presence of languages other than English. The proportion of nonEnglish webpages is on the increase, with $90 \%$ of Japanese webpages now in Japanese. It would seem that "The Web is increasingly reflecting the distributing of language presence in the real world, and there is a steadily growing set of sites which provide the evidence" (231). Remarkably, Crystal estimates that "over a quarter of the world's languages have some sort of Internet presence now" (233) - an encouraging sign indeed!

Chapter Eight, New varieties, is an entirely new chapter, written for the second edition, with the aim of including two new aspects of language on the Internet: blogging and instant messaging. 

Internet. After describing the phenomenon Crystal considers the linguistic implications of blogging. His examples display "a style of writing which has never been seen in a public, printed form, outside of literature, and even there it would take an ingenious novelist indeed to capture its innocent spontaneity and unpredictable thematic direction" (244). It is a style which Crystal likens intriguingly to "the spontaneous letter-writing of the late Middle Ages [- a style] which gradually went out of public use once the standard language was institutionalized in manuals of grammar, punctuation and usage, beginning in the second half of the eighteenth century" (245).

Instant messaging of the MSN type has seen a similar increase since 2000. Linguistically, Crystal considers instant messaging closest to chat, but with some important differences. There is, for example, more "chunking" in instant messaging: a single message is often broken up into a series of "chunks". Another distinctive feature is the increased intimacy of the participants which "promotes a greater level of informality and typographical idiosyncrasy" (255). The chapter concludes with a brief discussion of the possible linguistic interest of Voice over Internet Protocol (or VoIP) in the years to come.

14 The ninth and last chapter, The linguistic future of the Internet, begins by acknowledging that "the transience of the technology" (257) will probably mean that much of the eight previous chapters will soon be out of date. Crystal then goes on briefly to consider possible developments which might engender further linguistic varieties worthy of discussion, before looking at the linguistic implications of automatic translation and texting (or SMS's). A section on Applied Internet linguistics looks at applications of the Internet in the field of foreign language teaching with virtual classrooms providing areas in which students may express themselves in the other language. Language and the Internet concludes with a section entitled Increasing the richness of language, in which the author returns to a number of apocalyptic predictions on the linguistic dangers of the Internet, quoted in chapter 1, to express the very opposite view: "I do not see the Internet being the death of language, but the reverse [...] I view each of the Netspeak situations as an area of huge potential enrichment for individual languages" (275). For Crystal, "Netspeak is a development of millennial significance [...] the biggest language revolution ever".

Language and the Internet is a fascinating book - a comprehensive introduction to the topic for the non-specialist and an excellent starting-point for anyone intending to pursue research in the field. The book includes a number of useful tables. However, I regretted the absence of illustrations. In some areas an annotated screenshot would have obviated the need for lengthy explanations regarding, for example, the screen layout of synchronous chatgroups or the different varieties of written language present on many webpages. It was notably in the passages describing the phenomena in question that the differences between the two editions was, I felt, most obvious, as the interfaces described had, in some cases, evolved significantly. These are, however, minor criticisms of an extremely stimulating study. Crystal writes engagingly - the reader is carried along by his intellectual curiosity and his refreshing fascination with the phenomena he encounters. To these he brings a remarkable linguistic culture, never neglecting the broader picture, taking constant care to situate Netspeak relative to the history of the English language and, indeed, of language in general. The bibliography is as complete as one could hope for in such a volume. All this, together 
with the originality and scope of Crystal's reflection means that Language and the Internet does indeed, as the author remarks, suggest "material for a thousand theses" (274).

\section{BIBLIOGRAPHY}

BERNERS-LEE T., Weaving the Web. London, Orion Business Books, 1999.

CHERNY L. Conversation and Community: Chat in a Virtual World, Stanford, CA, CSLI Publications, 1999.

CRYSTAL D. The Cambridge Encyclopedia of the English Language, $2^{\text {nd }}$ ed. Cambridge: Cambridge University Press, 2003.

GRICE H.P., "Logic and conversation", in COLE and MORGAN (eds.), Syntax and Semantics 3: speech acts, New York, Academic Press, 1975: 41-58.

HERRING S.C. (ed.), Computer-mediated Communication: Linguistic, Social and Cross-cultural Perspectives, Amsterdam, Benjamins, 1996a.

HERRING S.C., "Two variants of an electronic message scheme", in HERRING (ed.), 1996b: 81-106.

\section{NOTES}

1. This characterisation is drawn from material in the Cambridge Encyclopedia of the English Language (2003).

2. Famously stated in Grice (1975).

3. Herring (1996).

4. Cherny (1999).

5. Berners-Lee (1999).

\section{AUTHORS}

\section{GRAHAM RANGER}

Graham Ranger, Department of English, Université d'Avignon et des Pays de Vaucluse, France. Graham Ranger works as a senior lecturer at the Université d'Avignon et des Pays de Vaucluse where he teaches primarily English Linguistics and Translation. Among other things an on-line creative writing seminar for Masters students and a searchable database of linguistic problems have brought him into contact with some of the issues addressed in Language and the Internet. 